\title{
Screening of neuraminidase inhibitory activities of some medicinal plants traditionally used in Lingnan Chinese medicines
}

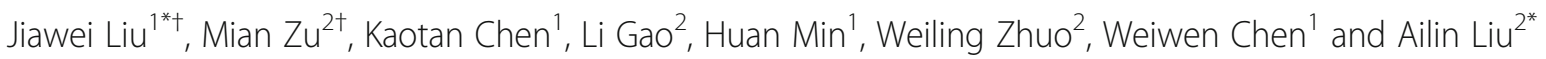

\begin{abstract}
Background: Neuraminidase (NA) is one of the key surface protein of the influenza virus, and has been established as a primary drug target for anti-influenza therapies. This study aimed to screen bioactive herbal extracts from some medicinal plants traditionally used in Lingnan Chinese Medicines by NA activity high-throughput screening assay.

Methods: One hundred ninety herbal extracts from 95 medicinal plants collected in Guangzhou were screened for their potential inhibitory activities against A ( $\mathrm{H} 1 \mathrm{~N} 1)$ influenza neuraminidase, and the most active extracts were further evaluated for their anti-influenza virus activities using virus-induced cytopathic effect (CPE).

Results: Among the tested 190 herbal extracts, 14 extracts inhibited significantly NA activity $\left(I_{50}<40 \mu \mathrm{g} / \mathrm{mL}\right)$, and the extracts 1-5, which were obtained from Amomurn villosum Lour, Melaphis chinensis (Bell) Baker, Sanguisorba officinalis and Flos Caryophylli, showed potent inhibitory activity against NA with IC 50 values ranging from 4.1 to 9. $6 \mu \mathrm{g} / \mathrm{mL}$. Moreover, the most bioactive extracts $1-5$ were found to protect MDCK cells from A (H1N1) influenza virus infection with very low cytotoxicity to the host cells ( $E C_{50}$ values ranged from 1.8 to $14.1 \mu \mathrm{g} / \mathrm{mL}$, $C C_{50}$ values ranged from 97.0 to $779.2 \mu \mathrm{g} / \mathrm{mL}$, SI values ranged from 14 to 438). In addition, quantitative RT-PCR analysis showed that the extracts 1-5 inhibited viral RNA synthesis in a dose-dependent manner.

Conclusion: We performed in vitro screening of anti-neuraminidase activities of herbal extracts from medicinal plants used in Lingnan Chinese Medicines, and the results indicate that some bioactive extracts are worth further studies to identify the bioactive components responsible for anti-influenza virus activities, to elucidate their modes of action and finally determine their clinical potentials.
\end{abstract}

Keywords: A (H1N1) influenza virus, Neuraminidase inhibitor, Anti-influenza agents, Medicinal plant, Lingnan Chinese medicines

\footnotetext{
* Correspondence: jiawei.liu@ymail.com; liuailin@imm.ac.cn

${ }^{\dagger}$ Equal contributors

${ }^{1}$ Ministry of Education Key Laboratory of Chinese Medicinal Resource from Lingnan, Research Center of Medicinal Plants Resource Science and Engineering, Guangzhou University of Chinese Medicine, 232 Waihuandong Road, Higher Education Mega Center, Guangzhou 510006, People's Republic of China

${ }^{2}$ Beijing Key Laboratory of Drug Target Research and Drug Screening, Institute of Material Medica, Chinese Academy Sciences and Peking Union Medical College, 1 Xian Nong Tan Street, Xicheng District, Beijing 100050, People's Republic of China
} International License (http://creativecommons.org/licenses/by/4.0/), which permits unrestricted use, distribution, and reproduction in any medium, provided you give appropriate credit to the original author(s) and the source, provide a link to the Creative Commons license, and indicate if changes were made. The Creative Commons Public Domain Dedication waiver (http://creativecommons.org/publicdomain/zero/1.0/) applies to the data made available in this article, unless otherwise stated. 


\section{Background}

Influenza virus causes an acute contagious respiratory tract infection, which is a major contributor to morbidity and mortality among human population. Historically pandemic flu has caused widespread human deaths, most notably the 1918 "Spanish Flu" (A/H1N1) which killed 25-50 million people worldwide [1]. Novel swineorigin influenza A (H1N1 subtype) virus identified in Mexico in 2009 emerges to spread rapidly worldwide via human-human transmission [2] and led to at least 17,798 deaths in 214 countries. Therefore, pandemic influenza A viruses such as the H1N1subtype becomes a serious global public health problem, which calls for more agents of anti-influenza therapies as possible.

Neuraminidase (NA) is an antigenic glycoprotein on the surface of influenza virus, which takes charge of catalyzing the cleavage of neuraminic acid residues to facilitate the detachment from the host cell surface at the end of the viral replication cycle and suppresses their self-aggregation of the virions [3, 4]. NA plays a critical role for virus replication and spread in infected tissues during infection, and has been well established as a primary drug target for anti-influenza therapies [5, 6]. Some potent NA inhibitors, including oseltamivir, zanamivir, laninamivir and peramivir, have been designed and applied in clinical treatments $[7,8]$. Unfortunately, resistance to these NA inhibitors has been extensively reported [9-11]. Therefore, there is a continuing need for developing novel NA inhibitors as anti-influenza agents. Medicinal plants may be a probable source for the discovery of natural NA inhibitors and might provide leads to develop the NA inhibitors [12].

In order to search for novel anti-influenza agents from natural resources, a library of 190 extracts of 95 medicinal plants traditionally used in Lingnan Chinese Medicines were screened for in vitro inhibitory activity against A (H1N1) influenza virus neuraminidase using highthroughput assay. The most active five extracts (1-5) were selected to further study their action upon the replication of influenza viruses using cytopathic effect (CPE) reduction assay and quantitative RT-PCR analysis. The results showed that these herbal extracts significantly inhibited the NA activity and the replication of influenza viruses, and exhibited very low cytotoxicity to the host cells.

\section{Methods}

\section{Plant materials}

Ninety nine medicinal plants traditionally used in Lingnan Chinese Medicines were collected in Guangzhou in 2009. The identity of the plants samples was verified by Dr. Guangtian Peng (Guangzhou University of Chinese Medicine). Voucher specimens of these materials were deposited for references in the Research Center of Medicinal Plants Resource Science and Engineering,
Guangzhou University of Chinese Medicine. The samples were stored in the shade at room temperature and pulverized before use.

\section{Standard extraction preparation}

Dried powdered plants (100 g) were extracted with ethyl acetate (EtOAc, $250 \mathrm{~mL} \times 3)$ and methanol $(\mathrm{MeOH}$, $250 \mathrm{~mL} \times 3$ ) by ultrasound wave at $40 \mathrm{kHz}$ and $400 \mathrm{~W}$ at $45{ }^{\circ} \mathrm{C}$ for $30 \mathrm{~min}$, the filtrates were evaporated under vacuum at $45{ }^{\circ} \mathrm{C}$ to give the EtOAc and $\mathrm{MeOH}$ extracts, respectively. A total of 190 herbal extracts were obtained. A stock solution for each extract was prepared by dissolution to dimethyl sulfoxide (DMSO), $50 \mathrm{mg}$ of each extract was suspended in $1 \mathrm{ml}$ of DMSO ensuing stock concentration of $50 \mu \mathrm{g} / \mu \mathrm{L}$. The solutions were filtered by using $0.22 \mu \mathrm{m}$ filters, and stored at $-20{ }^{\circ} \mathrm{C}$. The concentration of DMSO in test dilutions was restricted to no more than $0.5 \%(v / v)$ to minimize potential effects of the solvent on enzyme activity and cell growth.

\section{Neuraminidase, virus and cells}

The human influenza virus strains A/PR/8/34 (H1N1) was kindly provided by China Centers for Disease Control, and was used as the source of NA; Madin-Darby canine kidney (MDCK) and A549 cell lines were obtained from the National Center for Pharmaceutical Screening, Institute of Materia Medica, Chinese Academy of Medical Sciences. Madin-Darby canine kidney (MDCK) cells were grown in Dulbecco's modified Eagle medium (DMEM) containing $10 \%$ fetal bovine serum (FBS) at $37{ }^{\circ} \mathrm{C}$ and $5 \% \mathrm{CO}_{2}$ atmosphere. MDCK cells were used for virus infection, and were washed with PBS buffer before infection. 2' -(4-methylunbelliferyl)- $\alpha$ - $D$-acetyl-neuraminic acid (MUNANA), 2(N-Morpholino)-ethanesulfonic acid (MES) and 3-[4,5-dimethyl-thiazol-2-yl]-2,5-diphenyl tetrazolium bromide (MTT) were purchased from Sigma. DMEM, FBS, and $0.25 \%$ trypsin-EDTA were purchased from Gibco. Ribavirin with purity more than $98 \%$, and zanamivir with purity more than 98\% were purchased from Sigma (Lot\#020 M4003) and Full Land international trade company in Shanghai of China (Lot\#091209-005LY), respectively. They were used as references in NA and CPE inhibition assays.

\section{In vitro screening of plant extracts for NA activity}

Inhibition of influenza virus NA activity was determined by a standard fluorimetric method [13, 14] using4methylumbelliferyl- $\alpha-\mathrm{D}-\mathrm{N}$-acetyl-neuraminate (MUNANA) (Sigma) as substrate, in 96-well microplates. The reaction mixture containing the extracts or compounds, and NA enzyme in MES buffer (32.5 mM) and calcium chloride (4 mM, pH 6.5) was incubated for $60 \mathrm{~min}$. After incubation, the reaction was terminated by adding $\mathrm{NaOH}(34 \mathrm{mM})$. Fluorescence intensity $(\mathrm{M})$ was quantified with excitation wavelength at $360 \mathrm{~nm}$ and emission wavelength at $450 \mathrm{~nm}$. 
Percentage inhibition was calculated relative to a blank reaction mixture (solvent control) containing virus $\mathrm{NA}$ and solvent $\left(\%\right.$ Inhibition $\left.=\left[1-\left(\mathrm{M}_{\text {extract }} / \mathrm{M}_{\text {control }}\right)\right] \times 100\right)$. The $50 \%$ inhibitory concentration $\left(\mathrm{IC}_{50}\right)$ was defined as the concentration of NA inhibitor necessary to reduce NA activity by $50 \%$ relative to a blank reaction mixture. $\mathrm{IC}_{50}$ values displayed represent the mean of three individual determinations each performed in triplicate assays. Zanamivir (Sigma) was used as the reference compound.

\section{Cytotoxicity assay}

The cytotoxicity of medicinal plant extracts was determined with the MTT (Sigma) method as described previously [15]. Briefly, different concentrations of the extracts and compounds were added to each well of a 96well culture plate containing a confluent cell monolayer in triplicate, blank medium was used as the control. After incubation at $37{ }^{\circ} \mathrm{C}$ in an atmosphere of $5 \% \mathrm{CO}_{2}$ for $72 \mathrm{~h}$, $12 \mu \mathrm{L}$ of MTT solution $(5 \mathrm{mg} / \mathrm{ml}$ in phosphate buffered saline) was added to each well. The plate was further incubated at $37{ }^{\circ} \mathrm{C}$ for $3 \mathrm{~h}$ to allow formation of formazan product. After removing the medium, $100 \mu \mathrm{L}$ of DMSO was added to dissolve the formazan crystals. After $15 \mathrm{~min}$, the contents of the wells were homogenized on a microplate shaker. The optical densities (OD) were then determined by measuring absorbance with a microplate spectrophotometer at a wavelength of $540 \mathrm{~nm}$ and a reference wavelength of $620 \mathrm{~nm}$. The median cytotoxic concentration $\left(\mathrm{CC}_{50}\right)$ was calculated as the concentration of the constituent that reduced the viable cells to $50 \%$ of the untreated control. The maximal non-cytotoxic concentration (MNCC) was defined as the maximal concentration of the sample that did not exert a cytotoxic effect and resulted in more than $90 \%$ viable cells.

\section{CPE reduction assay}

The anti-viral activity of the extracts was measured by a virus-induced cytopathic effect (CPE) reduction assay as described previously [14, 16]. Briefly, $100 \mu \mathrm{L}$ of virus suspension of 200 tissue culture infective dose $\left(\mathrm{TCID}_{50} / \mathrm{mL}\right)$ was added to each well of a 96well culture plate containing confluent a MDCK cells monolayer. After incubation at $37{ }^{\circ} \mathrm{C}$ for $2 \mathrm{~h}$, the virus solution was removed, and $100 \mu \mathrm{L}$ of serial dilutions of the extracts and ribavirin were added to each well of the 96-well culture plates, using the maximal noncytotoxic concentration (MNCC) as the highest concentration. The plates were incubated at $37{ }^{\circ} \mathrm{C}$ in a humidified $5 \%$ $\mathrm{CO}_{2}$ atmosphere for $48 \mathrm{~h}$, and then the CPE was assessed. The virus-induced CPE was scored as follows: $0=$ no $\mathrm{CPE}$, $1=0-25 \%$ CPE, $2=25 \%-50 \%$ CPE, $3=50 \%-75 \%$ CPE, and $4=75 \%-100 \%$ CPE. Apart from test group, there were control group (treated with FBS-free medium instead of extracts and virus) and model group (treated with FBS-free medium and virus instead of extracts and virus). The CPE inhibition ratios were calculated using the equation: CPE inhibition $\%=100-\left[\left(\mathrm{OD}_{\text {test }}-\mathrm{OD}_{\text {control }}\right)^{*} 100 / \quad\left(\mathrm{OD}_{\text {model }}{ }^{-}\right.\right.$ $\left.\mathrm{OD}_{\text {control }}\right)$. The $\mathrm{OD}_{\text {test }}, \mathrm{OD}_{\text {model }}$, and $\mathrm{OD}_{\text {control }}$ mean the optical density of test group, model group, and control group, respectively. At least three independent experiments with three parallel experiments were performed to determine the mean and SD value.

\section{Measurement of viral RNA synthesis by quantitative and reverse transcription PCR ( $\mathrm{PPCR}$ )}

A549 cells were grown in RPMI1640 to about 90\% confluence and were infected with influenza virus $\mathrm{A} / \mathrm{PR} / 8 / 34$ (H1N1) influenza virus at $100 \mathrm{TCID}_{50}$, followed by administration of test extracts for $5 \mathrm{~h}$. To determine the expression level of hemagglutinin (HA) gene mRNA of influenza virus, cells were harvested and the total RNA was extracted by TRIzol (Invitrogen) according to the manufacture's instruction. The primer sequences which were designed by Primer-BLAST from NCBI for quantitative real-time PCR of influenza virus were 5'-CCTGCTCGAAGACAGCCACAACG-3' (sense) and 5'-TTCCCAAGAGCCATCCGG CGA-3' (antisense). The GAPDH were used as internal control of cellular RNAs, with primer sequence of $5^{\prime}$ - TGC TCCGAAGGGTGGCCCTTA-3' (sense) and 5' - TGCGT GTTTCCAGAGCCGTGC-3'(antisense). The total RNA was reverse transcribed into cDNA using the TransScript First-Strand cDNA Synthesis SuperMix (TransGen Biotech, Beijing, China). The cDNA was used as template for realtime PCR conducted by SsoFast EvaGreen PCR $2 \times$ master mix (Bio-Rad) using CFX 96 Realtime PCR system (Bio Rad location) according to the manufacture's protocol. The data was analyzed using the mode for normalised expression $\left(2^{-\Delta \Delta \mathrm{Cq}}\right)$.

\section{Statistical analysis}

Statistical analysis was performed using the Student's unpaired t-test. The results were presented as mean \pm S.D. $(n=3) . " p<0.05$ and $" p<0.001$ indicate a statistically significant difference as compared to the untreated control.

\section{Results}

NA has been validated as one of the most important targets to screen the drugs of anti-influenza virus. We first examined the ability of 190 organic extracts from 95 medicinal plants to inhibit NA activity by in vitro screening assay. Zanamivir was used as a positive control, its $\mathrm{IC}_{50}$ value to NA inhibition was $0.05 \mu \mathrm{g} / \mathrm{mL}$. 14 extracts were found to effectively inhibit the NA activity at the concentration of $40 \mu \mathrm{g} / \mathrm{mL}$. Among them, 5 extracts exhibited potent inhibition of NA activity, 9 extracts exhibited moderate NA inhibitory activity with $\mathrm{IC}_{50}$ values ranged from 4.1 to $37.3 \mu \mathrm{g} / \mathrm{mL}$. The 
Table 1 Inhibitory activities of Chinese herbs extract on A(H1N1) influenza virus neuraminidase

\begin{tabular}{|c|c|c|c|c|c|c|}
\hline No. & Positive control and Botanical name & Botanical part & Extract & Inhibition (\%) ${ }^{a}$ & $I C_{50}^{b}$ & Voucher No. \\
\hline- & Zanamivir & - & - & 99.8 & 0.05 & - \\
\hline 1 & Melaphis chinensis (Bell)Baker & cecidium & $\mathrm{MeOH}$ & 103.6 & 4.1 & МCB091101 \\
\hline 2 & Amomurn villosum Lour. & fruit & $\mathrm{MeOH}$ & 92.2 & 4.9 & CG20080829 \\
\hline 3 & Sanguisorba officinalis $L$. & root & $\mathrm{MeOH}$ & 100.8 & 5.1 & SOL091101 \\
\hline 4 & Melaphis chinensis (Bell)Baker & cecidium & EtOAc & 99.3 & 5.3 & МСB091101 \\
\hline 5 & Flos Caryophylli & flowers & $\mathrm{MeOH}$ & 94.1 & 9.1 & SA091101 \\
\hline 6 & Areca catechu Linn & fruit & $\mathrm{MeOH}$ & 85.1 & 19.3 & ACL091101 \\
\hline 7 & Artemisia capillaries Thunb & whole plant & $\mathrm{MeOH}$ & 91.3 & 19.4 & АСТ091101 \\
\hline 8 & Terminalia chebula Retz & fruit & EtOAc & 78.4 & 20.3 & TCR091101 \\
\hline 9 & Duchesnea indica (Andr.) Focke & whole plant & $\mathrm{EtOAc}$ & 69.1 & 23.3 & DIF091101 \\
\hline 10 & Terminalia chebula Retz. & fruit & $\mathrm{MeOH}$ & 68 & 24.3 & TCR091101 \\
\hline 10 & Murraya exotica L. & stem and leaves & $\mathrm{MeOH}$ & 65.7 & 28.9 & MEL091101 \\
\hline 11 & Geranium carolinianum L. & whole plant & $\mathrm{MeOH}$ & 64.8 & 28.9 & GCL091101 \\
\hline 12 & Polygonum cuspidatum & rhizome & EtOAc & 63.9 & 29.8 & PC091101 \\
\hline 13 & Saposhnikovia divaricata (Turez.) Schischk. & root & EtOAc & 53.1 & 37.3 & SDS091101 \\
\hline 14 & Callicarpa formosana Rolfe & fruit & $\mathrm{MeOH}$ & 47.9 & $N T^{d}$ & CFR091103 \\
\hline 15 & Gardenia jasminoides Ellis & fruit & $\mathrm{MeOH}$ & 46.6 & NT & GJE091101 \\
\hline 16 & Duchesnea indica (Andr.) Focke & whole plant & EtOAc & 46.1 & NT & DIF091101 \\
\hline 17 & Rosa laevigata Michx. & stem and leaves & $\mathrm{EtOAC}$ & 45.8 & NT & RLM091103 \\
\hline 18 & Euphorbia humifusa Willd. ex Schlecht. & whole plant & $\mathrm{MeOH}$ & 43.9 & NT & EHW091101 \\
\hline 19 & Litchi chinensis Sonn. & seed & $\mathrm{EtOAC}$ & 43.9 & NT & LCS091101 \\
\hline 20 & Punica granatum L. & fruit peel & $\mathrm{MeOH}$ & 43.4 & NT & PGL091101 \\
\hline 21 & Scutellaria baicalensis Georgi & root & $\mathrm{EtOAC}$ & 41.3 & NT & SBG091101 \\
\hline 22 & Amomum villosum Lour. & fruit & $\mathrm{EtOAc}$ & 40.5 & NT & CG20080829 \\
\hline 23 & Geranium carolinianum L. & whole plant & EtOAc & 40.1 & NT & GCL091101 \\
\hline 24 & Isatis indigotica Fort & stem and leaves & EtOAc & 40.1 & NT & IIF091103 \\
\hline 25 & Onosma gmelinii Ledeb & root & EtOAc & 40 & NT & OGL091101 \\
\hline 26 & Houttuynia cordata Thunb & whole plant & EtOAc & 38.5 & NT & НСТ091101 \\
\hline 27 & Altingia chinensis (Champ.) Oliver ex Hance & stem and leaves & EtOAc & 37.3 & NT & ACO091103 \\
\hline 28 & Pogostemon cablin (Blanco) Bent. & whole plant & $\mathrm{EtOAC}$ & 36.7 & NT & PCB091101 \\
\hline 29 & Polygonum cuspidatum & rhizome & $\mathrm{MeOH}$ & 36.1 & NT & PC091101 \\
\hline 30 & Punica granatum $L$. & fruit peel & EtOAc & 35.5 & NT & PGL091101 \\
\hline 31 & Rosa laevigata Michx. & stem and leaves & $\mathrm{MeOH}$ & 34.4 & NT & RLM091103 \\
\hline 32 & Dianella ensifolia (Linn.) Redouté & fruit & EtOAc & 31.5 & NT & DER091103 \\
\hline 33 & Elsholtzia ciliata (Thunb.) Hyland. & whole plant & $\mathrm{MeOH}$ & 31.3 & NT & ECH091101 \\
\hline 34 & Atractylodes Lancea (Thunb) DC. & root & EtOAc & 30.4 & NT & ALD091101 \\
\hline 35 & Cynanchum otophyllum Schneid. & root & EtOAc & 29.3 & NT & cos091101 \\
\hline 36 & Homalocladium platycladum (F. Muell.) Bailey & whole plant & $\mathrm{MeOH}$ & 29.1 & NT & HPB091101 \\
\hline 37 & Cinnamomum cassia Presl & branch & $\mathrm{MeOH}$ & 28.9 & NT & ССР091101 \\
\hline 38 & Elsholtzia ciliata (Thunb.) Hyland. & whole plant & EtOAc & 28.1 & NT & ЕСР091101 \\
\hline 39 & Sarcandra glabra (Thunb.) Nakai & stem and leaves & EtOAc & 26.8 & NT & SGN091103 \\
\hline 40 & Altingia chinensis (Champ.) Oliver ex Hance & stem and leaves & $\mathrm{MeOH}$ & 25.8 & NT & ACO091103 \\
\hline 41 & Litchi chinensis Sonn. & seed & $\mathrm{MeOH}$ & 25.5 & NT & LCS091101 \\
\hline 42 & Phellodendron chinense Schneid & bark & EtOAC & 25.4 & NT & PCS091101 \\
\hline
\end{tabular}


Table 1 Inhibitory activities of Chinese herbs extract on A(H1N1) influenza virus neuraminidase (Continued)

\begin{tabular}{|c|c|c|c|c|c|c|}
\hline No. & Positive control and Botanical name & Botanical part & Extract & Inhibition (\%) & $I C_{50}^{b}$ & Voucher No. \\
\hline 43 & Euphorbia humifusa Willd. ex Schlecht. & whole plant & EtOAc & 23.6 & NT & EHW091101 \\
\hline 44 & Glycyrrhiza uralensis Fisch. & rhizome & EtOAc & 23.1 & NT & GUF091101 \\
\hline 45 & Woodwardia japonica (L. f.) Sm. & rhizome & $\mathrm{MeOH}$ & 23 & NT & WJS091101 \\
\hline 46 & Ardisia japonica (Thunb) Blume & whole plant & $\mathrm{MeOH}$ & 22.7 & NT & AJB091101 \\
\hline 47 & Cinnamomum cassia Presl & branch & EtOAc & 22.7 & NT & ССР091101 \\
\hline 48 & Equisetum hyemale L. & whole plant & EtOAc & 22.1 & NT & EHL091101 \\
\hline 49 & Fraxinus rhynchophylla Hance & bark & EtOAc & 22.1 & NT & FRH091101 \\
\hline 50 & Ardisia japonica (Thunb.) Blume & whole plant & EtOAc & 21.7 & NT & AJB091101 \\
\hline 51 & Andrographis paniculata (Burm. f.) Nees & whole plant & EtOAc & 20.8 & NT & APN091101 \\
\hline 52 & Punica granatum Linn. & stem & EtOAc & 20.2 & NT & AGL091103 \\
\hline 53 & Syzygium aromaticum & flowers & EtOAc & 19.5 & NT & SA091101 \\
\hline 54 & Artemisia capillaris Thunb. & whole plant & EtOAc & 19.2 & NT & АСТ091101 \\
\hline 55 & Nepeta cataria L. & whole plant & $\mathrm{MeOH}$ & 18.9 & NT & NCL091101 \\
\hline 56 & Lonicera japonica Thunb. & flowers & $\mathrm{MeOH}$ & 18 & NT & AJT091101 \\
\hline 57 & Woodwardia japonica (L. f.) Sm. & rhizome & EtOAc & 17.9 & NT & WJS091101 \\
\hline 58 & Nepeta cataria $L$. & whole plant & EtOAc & 17.4 & NT & NCL091101 \\
\hline 59 & Dendranthema indicum (L.) Des Moul. & flowers & EtOAc & 16.5 & NT & DID091101 \\
\hline 60 & Senecio scandens Buch. -Ham. ex D. Don & whole plant & $\mathrm{MeOH}$ & 16.3 & NT & SSB091101 \\
\hline 61 & Onosma gmelinii Ledeb & root & $\mathrm{MeOH}$ & 15.9 & NT & OGL091101 \\
\hline 62 & Evodia rutaecarpa (Juss.) Benth. & fruit & $\mathrm{MeOH}$ & 15.5 & NT & ERB091101 \\
\hline 63 & Ligusticum chuanxiong Hort. & root & $\mathrm{MeOH}$ & 15.5 & NT & LCH091101 \\
\hline 64 & Atractylodes Lancea (Thunb.) DC. & root & $\mathrm{MeOH}$ & 15.2 & NT & ALD091101 \\
\hline 65 & Punica granatum L. & leaves & $\mathrm{MeOH}$ & 15 & NT & PGL091101 \\
\hline 66 & Artemisia indices Willd. & leaves & $\mathrm{MeOH}$ & 14.8 & NT & AlW091101 \\
\hline 67 & Serissa japonica (Thunb.) Thunb. & stem and leaves & EtOAc & 14.8 & NT & SJT091101 \\
\hline 68 & Prunella vulgaris $L$. & whole plant & $\mathrm{MeOH}$ & 14.1 & NT & PVL091101 \\
\hline 69 & Dicliptera chinensis (L.) Juss. & whole plant & $\mathrm{MeOH}$ & 14 & NT & DCJ091101 \\
\hline 70 & Glycyrrhiza uralensis Fisch. & rhizome & $\mathrm{MeOH}$ & 13.7 & NT & GUF091101 \\
\hline 71 & Platycladus orientalis (L.) Franco & leaves & EtOAc & 13.4 & NT & POF091101 \\
\hline 72 & Angelica dahurica (Fisch. ex Hoffm.) Benth. & root & $\mathrm{MeOH}$ & 13.3 & NT & ADB091101 \\
\hline 73 & Sarcandra glabra (Thunb.) Nakai & stem and leaves & $\mathrm{MeOH}$ & 13.3 & NT & SGN091101 \\
\hline 74 & Cynanchum otophyllum Schneid. & root & $\mathrm{MeOH}$ & 13 & NT & COS091101 \\
\hline 75 & Clerodendrum fortunatum Linn. & stem and leaves & EtOAc & 12.5 & NT & CFL091101 \\
\hline 76 & Scutellaria baicalensis Georgi & root & $\mathrm{MeOH}$ & 12.2 & NT & SBG091101 \\
\hline 77 & Sophora flavescens Alt. & root & $\mathrm{MeOH}$ & 11.6 & NT & SFA091101 \\
\hline 78 & Paris verticillata M.Bieb. & rhizome & EtOAc & 11.4 & NT & PVM091101 \\
\hline 79 & Semiaquilegia adoxoides (DC.) Makino & whole plant & EtOAc & 11.4 & NT & SAM091101 \\
\hline 80 & Magnolia liliflora Desr. & flowers & EtOAc & 11.3 & NT & MLD091101 \\
\hline 81 & Albizia julibrissin Durazz. & flowers & $\mathrm{MeOH}$ & $N A^{c}$ & NT & AJD091101 \\
\hline 82 & Albizia julibrissin Durazz. & flowers & EtOAc & NA & NT & AJD091101 \\
\hline 83 & Andrographis paniculata (Burm. f.) Nees & whole plant & $\mathrm{MeOH}$ & NA & NT & APN091101 \\
\hline 84 & Angelica dahurica (Fisch. ex Hoffm.) Benth. & root & EtOAc & NA & NT & ADB091101 \\
\hline 85 & Arctium lappa L. & seed & $\mathrm{MeOH}$ & NA & NT & ALL091101 \\
\hline 86 & Arctium lappa $L$. & seed & EtOAc & NA & NT & ALL091101 \\
\hline
\end{tabular}


Table 1 Inhibitory activities of Chinese herbs extract on A(H1N1) influenza virus neuraminidase (Continued)

\begin{tabular}{|c|c|c|c|c|c|c|}
\hline No. & Positive control and Botanical name & Botanical part & Extract & Inhibition (\%) & $I C_{50}^{b}$ & Voucher No. \\
\hline 87 & Areca catechu Linn & fruit & EtOAc & NA & NT & ACL091101 \\
\hline 88 & Artemisia argyi Levl. et Van. & leaves & $\mathrm{MeOH}$ & NA & NT & AAL091101 \\
\hline 89 & Artemisia argyi Levl. et Van. & leaves & $\mathrm{EtOAC}$ & NA & NT & AAL091101 \\
\hline 90 & Artemisia carvifolia Buch. -Ham. ex Roxb. & whole plant & EtOAc & NA & NT & ACB091101 \\
\hline 91 & Artemisia carvifolia Buch. -Ham. ex Roxb. & whole plant & $\mathrm{MeOH}$ & NA & NT & ACB091101 \\
\hline 92 & Artemisia indices Willd. & leaves & EtOAc & NA & NT & AlW091103 \\
\hline 93 & Bidens pilosa Linn. & whole plant & EtOAc & NA & NT & BPL091103 \\
\hline 94 & Bidens pilosa Linn. & whole plant & $\mathrm{MeOH}$ & NA & NT & BPL091103 \\
\hline 95 & Bupleurum tenue Buch-Ham. ex D. Don & root & EtOAc & NA & NT & BTB091101 \\
\hline 96 & Bupleurum tenue Buch-Ham. ex D. Don & root & $\mathrm{MeOH}$ & NA & NT & BTB091101 \\
\hline 97 & Callicarpa formosana Rolfe & fruit & EtOAc & NA & NT & CFR091103 \\
\hline 98 & Clerodendrum fortunatum Linn. & stem and leaves & $\mathrm{MeOH}$ & NA & NT & CFL091103 \\
\hline 99 & Clinopodium megalanthum & seed & EtOAc & NA & NT & CMC091101 \\
\hline 100 & Clinopodium megalanthum & seed & $\mathrm{MeOH}$ & NA & NT & CMC091101 \\
\hline 101 & Crataegus pinnatifida Bge. & fruit & $\mathrm{MeOH}$ & NA & NT & CPB091101 \\
\hline 102 & Crataegus pinnatifida Bge. & fruit & $\mathrm{EtOAc}$ & NA & NT & CPB091101 \\
\hline 103 & Dendranthema indicum (L.) Des Moul. & flowers & $\mathrm{MeOH}$ & NA & NT & DID091101 \\
\hline 104 & Dendranthema morifolium (Ramat.) Tzvel. & flowers & EtOAc & NA & NT & DMT091101 \\
\hline 105 & Dendranthema morifolium (Ramat.) Tzvel. & flowers & $\mathrm{MeOH}$ & NA & NT & DMT091101 \\
\hline 106 & Dianella ensifolia (Linn.) Redouté & fruit & $\mathrm{MeOH}$ & NA & NT & DER091103 \\
\hline 107 & Dicliptera chinensis (L.) Juss. & whole plant & EtOAc & NA & NT & DCJ091103 \\
\hline 108 & Duchesnea indica (Andr.) Focke & whole plant & $\mathrm{MeOH}$ & NA & NT & DIF091103 \\
\hline 109 & Epaltes australis Less. & whole plant & EtOAc & NA & NT & EAL091101 \\
\hline 110 & Epaltes australis Less. & whole plant & $\mathrm{MeOH}$ & NA & NT & EAL091101 \\
\hline 111 & Equisetum hyemale $L$. & whole plant & $\mathrm{MeOH}$ & NA & NT & EHL091101 \\
\hline 112 & Euchresta japonica Hook. f. ex Regel & root & EtOAc & NA & NT & EJH091101 \\
\hline 113 & Euchresta japonica Hook. f. ex Regel & root & $\mathrm{MeOH}$ & NA & NT & EJH091101 \\
\hline 114 & Eupatorium catarium Veldkamp & whole plant & $\mathrm{MeOH}$ & NA & NT & ECV091103 \\
\hline 115 & Eupatorium catarium Veldkamp & whole plant & EtOAc & NA & NT & ECV091103 \\
\hline 116 & Eupatorium fortunei Turcz. & whole plant & EtOAc & NA & NT & EFT091101 \\
\hline 117 & Eupatorium fortunei Turcz. & whole plant & $\mathrm{MeOH}$ & NA & NT & EFT091101 \\
\hline 118 & Eupolyphaga seu Steleophaga & insect & EtOAc & NA & NT & ESS091101 \\
\hline 119 & Eupolyphaga seu Steleophaga & insect & $\mathrm{MeOH}$ & NA & NT & ESS091101 \\
\hline 120 & Evodia rutaecarpa (Juss.) Benth. & fruit & EtOAc & NA & NT & ERB091101 \\
\hline 121 & Ficus hirta Vahl & leaves & $\mathrm{MeOH}$ & NA & NT & FHV091101 \\
\hline 122 & Ficus hirta Vahl & leaves & $\mathrm{EtOAc}$ & NA & NT & FHV091101 \\
\hline 123 & Forsythia suspensa (Thunb.) Vahl & fruit & $\mathrm{MeOH}$ & NA & NT & FSV091101 \\
\hline 124 & Forsythia suspensa (Thunb.) Vahl & fruit & EtOAc & NA & NT & FSV091101 \\
\hline 125 & Fraxinus rhynchophylla Hance & bark & $\mathrm{MeOH}$ & NA & NT & FRH091101 \\
\hline 126 & Gardenia jasminoides Ellis & fruit & EtOAc & NA & NT & GJE091101 \\
\hline 127 & Homalocladium platycladum (F. Muell.) Bailey & whole plant & EtOAc & NA & NT & HPB091103 \\
\hline 128 & Homalomena occulta (Lour.) Schot & rhizome & $\mathrm{MeOH}$ & NA & NT & HOS091101 \\
\hline 129 & Homalomena occulta (Lour.) Schot & rhizome & EtOAc & NA & NT & HOS091101 \\
\hline 130 & Houttuynia cordata Thunb & whole plant & $\mathrm{MeOH}$ & NA & NT & НСТ091101 \\
\hline
\end{tabular}


Table 1 Inhibitory activities of Chinese herbs extract on A(H1N1) influenza virus neuraminidase (Continued)

\begin{tabular}{|c|c|c|c|c|c|c|}
\hline No. & Positive control and Botanical name & Botanical part & Extract & Inhibition (\%) & $I C_{50}^{b}$ & Voucher No. \\
\hline 131 & Ilex cornuta Lindl & stem & $\mathrm{MeOH}$ & NA & NT & ICL091103 \\
\hline 132 & Ilex cornuta Lindl & stem & EtOAc & NA & NT & ICL091103 \\
\hline 133 & Inula japonica Thunb. & flowers & $\mathrm{MeOH}$ & NA & NT & |JT091101 \\
\hline 134 & Inula japonica Thunb. & flowers & EtOAc & NA & NT & |JT091101 \\
\hline 135 & Isatis indigotica Fort & stem and leaves & $\mathrm{MeOH}$ & NA & NT & IIF091103 \\
\hline 136 & Ligusticum chuanxiong Hort. & root & EtOAc & NA & NT & LCH091101 \\
\hline 137 & Lobelia chinensis Lour. & whole plant & $\mathrm{MeOH}$ & NA & NT & LCH091101 \\
\hline 138 & Lobelia chinensis Lour. & whole plant & EtOAc & NA & NT & LCL091101 \\
\hline 139 & Lonicera confusa (Sweet) DC. & stem and leaves & $\mathrm{MeOH}$ & NA & NT & LCD091103 \\
\hline 140 & Lonicera confusa (Sweet) DC. & stem and leaves & EtOAc & NA & NT & LCD091103 \\
\hline 141 & Lonicera japonica Thunb. & flowers & EtOAc & NA & NT & LJT091101 \\
\hline 142 & Lonicera japonica Thunb. & stem and branch & $\mathrm{MeOH}$ & NA & NT & LJT091101 \\
\hline 143 & Lonicera japonica Thunb. & stem and branch & EtOAc & NA & NT & LJT091101 \\
\hline 144 & Lycium chinense Mill. & root bark & $\mathrm{MeOH}$ & NA & NT & LCM091101 \\
\hline 145 & Lycium chinense Mill. & Root bark & EtOAc & NA & NT & LCM091101 \\
\hline 146 & Magnolia liliflora Desr. & flowers & $\mathrm{MeOH}$ & NA & NT & MLD091101 \\
\hline 147 & Melia azedarach L. & bark & EtOAc & NA & NT & MAL091103 \\
\hline 148 & Melia azedarach L. & bark & $\mathrm{MeOH}$ & NA & NT & MAL091103 \\
\hline 149 & Murraya exotica L. & stem and leaves & EtOAc & NA & NT & MEL091103 \\
\hline 150 & Mussaenda pubescens Ait. f. & stem and leaves & EtOAc & NA & NT & MPA091103 \\
\hline 151 & Mussaenda pubescens Ait. f. & stem and leaves & $\mathrm{MeOH}$ & NA & NT & MPA091103 \\
\hline 152 & Paris verticillata M.Bieb. & rhizome & $\mathrm{MeOH}$ & NA & NT & PVM091101 \\
\hline 153 & Perilla frutescens (L.) Britt. & flowers & EtOAc & NA & NT & PFB091103 \\
\hline 154 & Perilla frutescens (L.) Britt. & flowers & $\mathrm{MeOH}$ & NA & NT & PFB091103 \\
\hline 155 & Peucedanum praeruptorum Dunn & root & EtOAc & NA & NT & PPD091101 \\
\hline 156 & Peucedanum praeruptorum Dunn & root & $\mathrm{MeOH}$ & NA & NT & PPD091101 \\
\hline 157 & Phellodendron chinense Schneid & bark & $\mathrm{MeOH}$ & NA & NT & PCS091101 \\
\hline 158 & Phytolacca acinosa Roxb. & root & EtOAc & NA & NT & PAR091101 \\
\hline 159 & Phytolacca acinosa Roxb. & root & $\mathrm{MeOH}$ & NA & NT & PAR091101 \\
\hline 160 & Pinellia ternata (Thunb.) Breit. & stem & $\mathrm{MeOH}$ & NA & NT & PTB091101 \\
\hline 161 & Pinellia ternata (Thunb.) Breit. & stem & EtOAc & NA & NT & PTB091101 \\
\hline 162 & Platycladus orientalis (L.) Franco & leaves & $\mathrm{MeOH}$ & NA & NT & POF091101 \\
\hline 163 & Pogostemon cablin (Blanco) Bent. & whole plant & $\mathrm{MeOH}$ & NA & NT & РCB091101 \\
\hline 164 & Prunella vulgaris $L$. & whole plant & EtOAc & NA & NT & PVL091101 \\
\hline 165 & Punica granatum $L$. & leaves & EtOAc & NA & NT & PGL091103 \\
\hline 166 & Punica granatum Linn. & stem & $\mathrm{MeOH}$ & NA & NT & PGL091103 \\
\hline 167 & Sanguisorba officinalis $L$. & root & EtOAc & NA & NT & SOL091101 \\
\hline 168 & Saposhnikovia divaricata (Trucz.) Schischk. & root & $\mathrm{MeOH}$ & NA & NT & SDS091101 \\
\hline 169 & Scaphium wallichii Shott \& Endl. & seed & $\mathrm{MeOH}$ & NA & NT & SWS091101 \\
\hline 170 & Scaphium wallichii Shott \& Endl. & seed & EtOAc & NA & NT & SWS091101 \\
\hline 171 & Semiaquilegia adoxoides (DC.) Makino & whole plant & $\mathrm{MeOH}$ & NA & NT & SAM091101 \\
\hline 172 & Senecio scandens Buch-Ham. ex D. Don & whole plant & EtOAc & NA & NT & SSB091101 \\
\hline 173 & Serissa japonica (Thunb.) Thunb. & stem and leaves & $\mathrm{MeOH}$ & NA & NT & SJT091103 \\
\hline 174 & Sophora flavescens Alt. & root & EtOAc & NA & NT & SFA091101 \\
\hline
\end{tabular}


Table 1 Inhibitory activities of Chinese herbs extract on A(H1N1) influenza virus neuraminidase (Continued)

\begin{tabular}{|c|c|c|c|c|c|c|}
\hline No. & Positive control and Botanical name & Botanical part & Extract & Inhibition (\%) $)^{a}$ & $I C_{50}^{b}$ & Voucher No \\
\hline 175 & Stemona japonica (BI.) Miq. & root & $\mathrm{MeOH}$ & NA & NT & SJM091101 \\
\hline 176 & Stemona japonica (BI.) Miq. & root & EtOAc & NA & NT & SJM091101 \\
\hline 177 & Strobilanthes cusia (Ness) W. Ktze. & stem and leaves & $\mathrm{MeOH}$ & NA & NT & SCW091101 \\
\hline 178 & Strobilanthes cusia (Ness) W. Ktze. & stem and leaves & EtOAc & NA & NT & SCW091101 \\
\hline 179 & Thlaspi arvense L. & whole plant & $\mathrm{MeOH}$ & NA & NT & TAL091103 \\
\hline 180 & Thlaspi arvense L. & whole plant & EtOAc & NA & NT & TAL091103 \\
\hline 181 & Turczaninovia fastigiata (Fisch.) DC. & flowers & $\mathrm{MeOH}$ & NA & NT & TFD091101 \\
\hline 182 & Turczaninovia fastigiata (Fisch.) DC. & flowers & EtOAc & NA & NT & TFD091101 \\
\hline 183 & Vitex trifolia $L$. & stem and leaves & EtOAc & NA & NT & VTL091103 \\
\hline 184 & Vitex trifolia $L$. & stem and leaves & $\mathrm{MeOH}$ & NA & NT & VTL091103 \\
\hline 185 & Wikstroemia indica (Linn.) C. A. Mey. & whole plant & $\mathrm{MeOH}$ & NA & NT & WIC091103 \\
\hline 186 & Wikstroemia indica (Linn.) C. A. Mey. & whole plant & EtOAc & NA & NT & WIC091103 \\
\hline 187 & Xanthium sibiricum Patrin ex Widder & fruit & EtOAc & NA & NT & XSP091103 \\
\hline 188 & Xanthium sibiricum Patrin ex Widder & fruit & $\mathrm{MeOH}$ & NA & NT & XSP091103 \\
\hline 189 & Zanthoxylum nitidum (Roxb.) DC. & root & $\mathrm{MeOH}$ & NA & NT & ZND091101 \\
\hline 190 & Zanthoxylum nitidum (Roxb.) DC. & root & EtOAc & NA & NT & ZND091101 \\
\hline
\end{tabular}

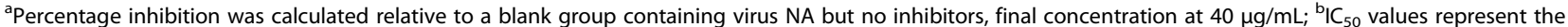
concentration that caused $50 \%$ NA enzyme activity loss, the average of at least three independent assays, $I C_{50}$ values are in $\mu \mathrm{g} / \mathrm{mL}$. ${ }^{\mathrm{s}}:$ not active; ${ }^{\mathrm{d}}:$ not test

bioactive extracts and their NA inhibition activity were summarized in Table 1 . The highest activity was demonstrated by $\mathrm{MeOH}$ extracts of Melaphis chinensis (1) and Amomurn villosum Lour (2) with $\mathrm{IC}_{50}=$ 4.1 and $4.9 \mu \mathrm{g} / \mathrm{mL}$, respectively. Significant activity with $\mathrm{IC}_{50}=5.0-10 \mu \mathrm{g} / \mathrm{mL}$ was also shown by $\mathrm{MeOH}$ extract of Sanguisorba officinalis (3), EtOAc extract of Melaphis chinensis (4) and $\mathrm{MeOH}$ extract of Flos Caryophylli (5). While other plant extracts (6-14) showed a moderate inhibitory activity on NA with the $\mathrm{IC}_{50}$ values ranging from 20.3 to $37.3 \mu \mathrm{g} / \mathrm{mL}$. These results demonstrated that these plant extracts possessed significant inhibitory activities against influenza virus NA and the most active extracts 1-5 were then selected to further study their effects on the replication of influenza virus.

To validate whether these extracts $\mathbf{1}-\mathbf{5}$ that exhibited NA inhibitory activity could protect host cells from influenza virus A (H1N1) infections, the CPE reduction assay was carried out in MDCK cells. The human influenza virus $\mathrm{A} / \mathrm{PR} / 8 / 34$ (H1N1) strain was used to infect MDCK cells. Cells were incubated in the presence or absence of the extracts 1-5, after $48 \mathrm{~h}$ of incubation, their CPE reduction activity on virus multiplication was then examined. As shown in Table 2, the extracts 1-5 could protect MDCK cells from the infection of influenza virus A (H1N1), exhibited a drastic reduction of influenza virus-induced $\mathrm{CPE}$. The $\mathrm{EC}_{50}$ values of the extracts 1-5 ranged from 1.8 to $14.1 \mu \mathrm{g} / \mathrm{mL}$, similar to the results obtained in NA assays. Among the five extracts, the $\mathrm{MeOH}$ extract (2) from the fruits of Amomurn villosum had excellent CPE activity with very low $\mathrm{EC}_{50}$ values of $1.8 \mu \mathrm{g} / \mathrm{mL}$, this is comparable to that of the positive compound ribavirin $(3.2 \mu \mathrm{g} / \mathrm{mL})$. The viability of MDCK cells incubated in the presence or absence of the extracts was evaluated by MTT assay, the $\mathrm{CC}_{50}$ values of the extracts $\mathbf{1 - 5}$ was found to be from 97.0 to $779.2 \mu \mathrm{g} / \mathrm{mL}$, suggesting that the extracts protected significantly host cells from influenza virus infection and did not exhibit considerable cytotoxicity against MDCK cells. The maximal non-cytotoxic concentration (MNCC) of the extracts $\mathbf{1 - 5}$ were found to be from 30 to $300 \mu \mathrm{g} / \mathrm{mL}$ in MDCK cells. Their therapeutic selective index (SI) in MDCK cells ranged from

Table 2 Inhibitory activity of Chinese herbs extracts (1-5) on $\mathrm{A}(\mathrm{H} 1 \mathrm{~N} 1)$ influenza virus by CPE assay

\begin{tabular}{lllll}
\hline Sample No. & $\mathrm{EC}_{50}^{\mathrm{a}}$ & $\mathrm{CC}_{50}^{\mathrm{b}}$ & $\mathrm{MNCC}^{\mathrm{c}}$ & $\mathrm{Sl}^{\mathrm{d}}$ \\
\hline 1 & 7.7 & 184.3 & 30 & 24 \\
2 & 1.8 & 779.2 & 300 & 438 \\
3 & 8.1 & 478.4 & 100 & 59 \\
4 & 7.2 & 97.0 & 30 & 14 \\
5 & 14.1 & 744.3 & 300 & 53 \\
Ribavirin & 3.2 & $>100$ & $\mathbf{e}^{\mathrm{e}}$ & $>31$ \\
Zanamivir & $>90.4$ & $>1506.0$ & $>301.2$ & 17 \\
\hline
\end{tabular}

${ }^{\mathrm{a}} \mathrm{EC}_{50}$ : Effective concentration required to protect $50 \%$ of cells; ${ }^{\mathrm{b}} \mathrm{CC}_{50}$ : Median (50\%) cytotoxic concentration in MDCK cells; ${ }^{C}$ MNCC: Maximal non-cytotoxic concentration in MDCK cells, values in $\mu \mathrm{g} / \mathrm{mL}^{\mathrm{d}}{ }^{\mathrm{d}}$ Sl:Selectivity index, $\mathrm{CC}_{50} /$ $\mathrm{EC}_{50}$. ${ }^{\mathrm{e}}$ : not test 
14 to 438 , and among of them, the SI value of A. villosum was highest on basis of its low cytotoxicity and its high CPE effect. These data demonstrated that the extracts 1-5 protected MDCK host cells from viral damage with very low toxicity. Thus, in agreement with that these extracts inhibited NA activities, the extracts 1-5 reduced host cell damage caused by the influenza virus A (H1N1) infection.

To further examine whether the protective effect of the extracts1-5 is related with the inhibition of influenza viral replication, total RNA was extracted and subjected to quantitative reverse-transcription PCR in the A/H1N1 virus-infected A549 cells. Our results showed that treatment with the extracts 1-5 for $5 \mathrm{~h}$ resulted in a substantial reduction in viral RNA expression level in a dose-dependent manner (Fig. 1). All extracts 1-5 at the high concentration $(30 \mu \mathrm{g} / \mathrm{mL})$ had significant inhibitory effects on viral RNA expression as compared with untreated control, even more powerful than ribavirin (Fig. 1). The extracts $\mathbf{2}-\mathbf{5}$ at medium concentration $(10 \mu \mathrm{g} / \mathrm{mL})$ also demonstrated significant inhibitory effects on viral RNA synthesis. Interestingly, the extracts $\mathbf{3}$ and $\mathbf{4}$ at low concentration of $3 \mu \mathrm{g} / \mathrm{mL}$ still significantly inhibited RNA synthesis of influenza viruses. These data indicate that the extracts 1-5 could inhibit significantly the replication of influenza viruses in cultures by RT-PCR analysis, which validated their anti-influenza viral activity obtained by CPE reduction assay.

\section{Discussion}

In the course of our screening of NA inhibitors for influenza virus A (H1N1), a total of 190 extracts of 95 medicinal plants traditionally used in Lingnan Chinese Medicines were submitted to in vitro screening for their NA inhibitory activities. Among of them, the organic extracts 1-5, obtained from Melaphis chinensis, Amomurn villosum, Sanguisorba officinalis and Flos Caryophylli, were found to significantly inhibit the NA activity $\left(\mathrm{IC}_{50}\right.$ $<10 \mu \mathrm{g} / \mathrm{mL}$, Table 1) and the replication of influenza virus in a dose-dependent manner (Fig. 1), and exhibited very low cytotoxicity to the host cells with the high selective index (SI) values ranging 14 to 438 (Table 2). Therefore, these Chinese herb extracts might contain bioactive components responsible for anti-influenza virus activity at non-toxic concentration and they could be a promising source of natural NA inhibitors.

It was demonstrated previously that the aqueous extracts of barks, leaves and galls of Melaphis chinensis have anti-influenza virus activity and some compounds such as gallotannins isolated from $M$. chinensis are responsible for the anti-influenza virus effect [17]. The presence of such compounds in our EtOAc and $\mathrm{MeOH}$ extracts of galls of $M$. chinensis may explain the biological activities seen in our screenings.

Flos Caryophylli also known as cloves, is considered acrid, warm and aromatic in Traditional Chinese Medicines for the treatment of stomachache, diarrhea and dental pain

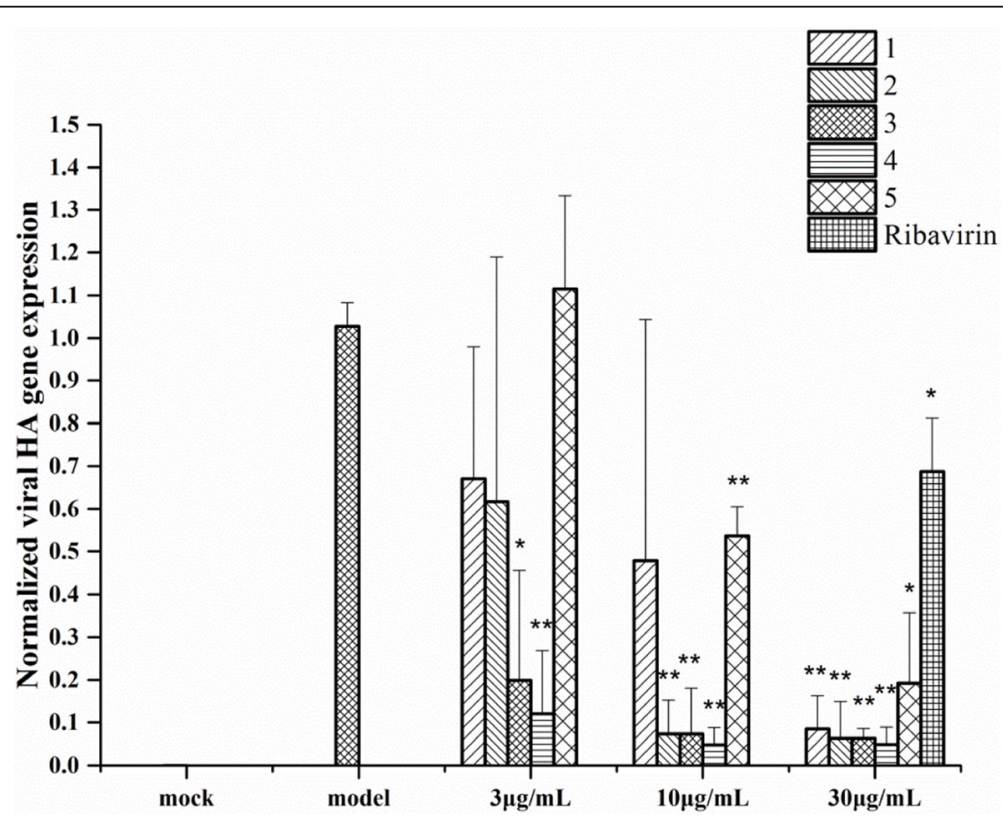

Fig. 1 Dose-dependent inhibitory effect of the extracts $\mathbf{1 - 5}$ on viral RNA synthesis. A549 cells were infected with 100 TCID 50 influenza H1N1 viruses and treated with different concentrations of the extracts $\mathbf{1 - 5}(3,10$ or $30 \mu \mathrm{g} / \mathrm{mL})$ and the DMSO $(0.03 \%)$ for 5 h. The total RNA was extracted and followed by GPCR analysis. To quantify the changes in gene expression, the $2^{-\triangle \Delta C(q)}$ method was used to calculate relative changes which were normalized to the GAPDH gene and the untreated control (model group, which was set to 1). Value calculated as Mean \pm SD of three independent tests, with ${ }^{*} p<0.05$ and ${ }^{* *} p<0.001$, respectively 
[18]. It was reported that the hot water extract of Flos Caryophylli have been shown to have anti-herpes virus, anti-hepatitis $\mathrm{C}$ virus and anti-cytomegalovirus activities in vitro and in vivo, and compounds such as ellagitannin and eugeniin were identified as the bioactive components with anti-virus properties [19]. In the present study, the $\mathrm{MeOH}$ extract of Flos Caryophylli showed $\mathrm{IC}_{50}$ value of $9.1 \mu \mathrm{g} / \mathrm{mL}$ towards NA and $\mathrm{EC}_{50}$ value of $14.1 \mu \mathrm{g} / \mathrm{mL}$ against influenza virus. In our latest phytochemical study on the $\mathrm{MeOH}$ extract of Flos Caryophylli [14], a bioassay-guided isolation led to identification of ten flavonoids, seven tannins and two chromones as NA inhibitors with $\mathrm{IC}_{50}$ values ranging from 8.4 to $94.1 \mu \mathrm{M}$. These polyphenolic constituents were found to protect MDCK cells from $\mathrm{A}(\mathrm{H} 1 \mathrm{~N} 1)$ influenza infections $\left(\mathrm{EC}_{50}=1.5-84.7 \mu \mathrm{M}\right)$ with very low cytotoxicity to the host cells $\left.\left(\mathrm{CC}_{50}=374.3-1266.9 \mu \mathrm{M}\right)\right)$, with selective index (SI) ranging from 7 to 297 [14].

The roots of $S$. officinalis (Rosaceae) are well-known Chinese herbs officially listed in the Chinese Pharmacopeia and have been used for the treatment of bleeding, diarrhea and burns. Early chemical studies showed that S. officinalis synthesize a variety of secondary metabolites, particularly polyphenols, triterpenoids, saponins and flavonoids with specific biological activities such as anti-asthmatic, antibacterial, anti-cancer and anti-inflammation [20-25]. A variety of flavonoids, saponins and polyphenols isolated from medicinal plant have been studied extensively and exhibited anti-influenza activities [12]. The $\mathrm{MeOH}$ extract of $S$. officinalis showed strong activities towards NA ( $\mathrm{IC}_{50}$ : $5.1 \mu \mathrm{g} / \mathrm{mL})$ and against influenza virus $\left(E_{50}: 8.1 \mu \mathrm{g} / \mathrm{mL}\right)$. The anti-influenza activity may be due to the presence of flavonoids and polyphenols in the $\mathrm{MeOH}$ fraction.

The fruits of $A$. villosum (Zingiberaceae) were consumed widely as popular cooking spices in East Asian countries and have been traditionally used as a medicine to treat various digestive disorders [26]. The volatile oils of the fruits of A. villosum were shown to be the major components and suggested to be responsible for the different biological activities such as analgesic, antioxidation and anti-inflammation [27]. In this study, the $\mathrm{MeOH}$ extract of the fruits of $A$. villosumwas shown to significantly inhibit NA activities $\left(\mathrm{IC}_{50}: 4.9 \mu \mathrm{g} / \mathrm{mL}\right)$ and protect the host cells from CPE damage $\left(\mathrm{EC}_{50}: 1.8 \mu \mathrm{g} /\right.$ $\mathrm{mL}$ ) without cytotoxicity, and its therapeutic selective index (SI) is 439 in MDCK cell culture.

In this study, we limit our study on EtOAc and $\mathrm{MeOH}$ extracts of medical plants since bioassay-guided isolation of neuraminidase inhibitors in aqueous extracts remains a challenging task for us. However, this may decrease the risk of false-positive results in the enzyme-based screening caused by some interfering components present within aqueous extracts. Future study will try to improve the screening methods on aqueous extracts that may also contain active components with anti-neuraminidase activity.

\section{Conclusion}

We carried out the in vitro screening of antineuraminidase activity of 190 herbal extracts from 95 medicinal plants traditionally used in Lingnan Chinese Medicines. Among the tested extracts, 5 extracts, obtained from Amomurn villosum, Melaphis chinensis, Sanguisorba officinalis and Flos Caryophylli, showed potent NA inhibitory activity. Comprehensive literature survey revealed that no study has been reported on the effects of the organic extracts of $A$. villosum and S. officinalis on antiinfluenza virus activities and small-molecule NA inhibitors from these extracts have not been chemically identified yet. Further studies are underway to isolate bioactive components of these extracts by bioassay-guided fractionation, and to explore their antiviral mechanisms and finally determine their clinical potentials.

\section{Abbreviations}

CPE: Cytopathic effect; HA: Haemagglutinin; HHDP: Hexahydroxydiphenoyl; MDCK: Madin-Darby canine kidney; MNCC: Maximal non-cytotoxic concentration; MTT: 3-[4,5-dimethyl-thiazol-2-yl]-2,5- diphenyl tetrazolium bromide; MUNANA: methylumbelliferyl-a-D-N-acetylneuraminate; NA: Neuraminidase; SI: Selectivity index.

\section{Acknowledgements \\ The authors would like to acknowledge all the fellows in Research Center of Medicinal Plants Resource Science and Engineering, Guangzhou University of Chinese Medicine for their great support and encouragement. We also thanks for their assistance in collecting medicinal plants from Jinxing Qiu, Guozheng He and Honghui Huang.}

\section{Funding}

This work was supported by China National Natural Science Foundation Grant (No.81373432), Guangzhou Science and Technology Program Grant (No. 2014 J4100118) to JL, National Great Science and Technology Major Projects (2012ZX09301002-2013HXW-11) and Beijing Natural Science

Foundation Grant (No. 7152103) to AL.

Availability of data and materials

The data sets used and /or analysed during the current study available from the corresponding authors on reasonable request.

Authors' contributions

$J \mathrm{~L}$ and $\mathrm{AL}$ conceived and designed the study. $J$, $K C$ and $H M$ collected the herbs and prepared the herbal extracts. MZ, LG, WZ and AL carried out herbal screening and anti-influenza virus studies. $J$, MZ and $A L$ analyzed data. $J$ wrote the manuscript. All authors read and approved the final manuscript.

Ethics approval and consent to participate Not applicable.

Consent for publication Not applicable.

Competing interests

The authors declare that they have no competing interests.

\section{Publisher's Note}

Springer Nature remains neutral with regard to jurisdictional claims in published maps and institutional affiliations. 
Received: 23 March 2017 Accepted: 15 March 2018

Published online: 20 March 2018

\section{References}

1. Ansart S, Pelat C, Boelle PY, Carrat F, Flahault A, Valleron AJ. Mortality burden of the 1918-1919 influenza pandemic in Europe. Influenza Other Respir Viruses. 2009;3(3):99-106.

2. Dawood FS, Jain S, Finelli L, Shaw MW, Lindstrom S, Garten RJ, et al. Emergence of a novel swine-origin influenza a (H1N1) virus in humans. N Engl J Med. 2009;360(25):2605-15.

3. Gong J, Xu W, Zhang J. Structure and functions of influenza virus neuraminidase. Curr Med Chem. 2007;14(1):113-22.

4. Yang J, Liu S, Du L, Jiang S. A new role of neuraminidase (NA) in the influenza virus life cycle: implication for developing NA inhibitors with novel mechanism of action. Rev Med Virol. 2016;26(4):242-50.

5. Jagadesh A, Salam AA, Mudgal PP, Arunkumar G. Influenza virus neuraminidase (NA): a target for antivirals and vaccines. Arc Virol. 2016; 161(8):2087-94.

6. Air GM, Ghate AA, Stray SJ. Influenza neuraminidase as target for antivirals. Adv Virus Res. 1999;54:375-02.

7. Shobugawa Y, Saito R, Sato I, Kawashima T, Dapat C, et al. Clinical effectiveness of neuraminidase inhibitors-oseltamivir, zanamivir, laninamivir, and peramivir for treatment of influenza a(H3N2) and a(H1N1) infection: an observational study in the 2010-2011 influenza season in Japan. J Infect Chemother. 2012;18:858-64.

8. Von Itzstein M. The war against influenza: discovery and development of sialidase inhibitors. Natr Rev Drug Discov. 2007;6(12):967-74.

9. Subbarao K, Joseph T. Scientific barriers to developing vaccines against avian influenza viruses. Nat Rev Immunol. 2007;7:267-78.

10. Thorlund K, Awad T, Boivin G, Thabane L. Systematic review of influenza resistance to the neuraminidase inhibitors. BMC Infec Dis. 2011;11:134.

11. Tamura D, DeBiasi RL, Okomo-Adhiambo M, Mishin VP, Campbell AP, et al. Emergence of multidrug-resistant influenza a(H1N1)virus variants in an immunocompromised child treated with oseltamivir and Zanamivir. J Infec Dis. 2015;212(8):1209-13.

12. Grienke U, Schmidtke M, von Grafenstein S, Kirchmair J, Liedl KR, Rollinger JM. Influenza neuraminidase: a druggable target for natural products. Nat Prod Rep. 2012;29(1):11-36.

13. Chen KT, Zhou WL, Liu JW, Zu M, He ZN, Du GH, et al. Active neuraminidase constituents of Polygonum cuspidatum against influenza a(H1N1) influenza virus. Zhongguo Zhong Yao Za Zhi. 2012;37(20):3068-73.

14. He Z, Lian W, Liu J, Zheng R, Xu H, Du G, Liu A. Isolation, structural characterization and neuraminidase inhibitory activities of polyphenolic constituents from Flos Caryophylli. Phytochem Lett. 2017:19:160-7.

15. Liu AL, Liu B, Qin HL, Lee SM, Wang YT, Du GH. Anti-influenza virus activities of flavonoids from the medicinal plant Elsholtzia rugulosa. Planta Med. 2008; 74(8):847-51.

16. Liu AL, Wang HD, Lee SM, Wang YT, Du GH. Structure-activity relationship of flavonoids as influenza virus neuraminidase inhibitors and their in vitro antiviral activities. Bioorg Med Chem. 2008;16(15):7141-7.

17. Kim HJ, Park,CJ. Composition for prevention of influenza viral infection comprising tannic acid, air filter comprising the same and air cleaning device comprising the filter. US Patent 9,119,814; 2015.

18. Chen G, Shen Y, Zhang M, Zhu Z, Wang D, Liu X, Ma S. Studies on warming the middle-jiao and analgesic effect of flos Caryophylli. Zhongguo Zhong Yao Za Zhi. 1991;16(7):429-32.

19. Kurokawa M, Hozumi T, Basnet $\mathrm{P}$, Nakano M, Kadota S, Namba T, et al. Purification and characterization of eugeniin as an anti-herpesvirus compound from Geum japonicum and Syzygium aromaticum. J Pharmacol Exp Ther. 1998;284(2):728-35.

20. Kang SY, Seo JK, Lim JW. Antiviral pentacyclic triterpenoids isolated from Sanguisorba officinalis roots against viral hemorrhagic septicemia virus and simultaneous quantification by LC-MS/MS. Planta Med. 2016;81(Suppl 1):1-81.

21. Yu T, Lee YJ, Yang HM, Han S, Kim JH, Lee $Y$, et al. Inhibitory effect of Sanguisorba officinalis ethanol extract on $\mathrm{NO}$ and $\mathrm{PGE}_{2}$ production is mediated by suppression of NF-kappaB and AP-1 activation signaling cascade. J Ethnopharmacol. 2011;134(1):11-7.

22. Lee NH, Lee MY, Lee JA, Jung DY, Seo CS, Kim JH, et al. Anti-asthmatic effect of Sanguisorba officinalis L. and potential role of heme oxygenase-1 in an ovalbumin-induced murine asthma model. Int J Mol Med. 2010;26(2): 201-8.
23. Kim TG, Kang SY, Jung KK, Kang JH, Lee E, Han HM, et al. Antiviral activities of extracts isolated from terminalis chebula Retz., Sanguisorba officinalis L., Rubus coreanus Miq. And Rheum palmatum L. against hepatitis B virus. Phytother Res. 2001:15(8):718-20.

24. Chen X, Shang F, Meng Y, Li L, Cui Y, Zhang M, et al. Ethanol extract of Sanguisorba officinalis $L$. inhibits biofilm formation of methicillinresistant Staphylococcus aureus in an Ica-dependent manner. J Dairy Sci. 2015;98(12):8486-91.

25. Liu MP, Liao M, Dai C, Chen JF, Yang CJ, Liu M, et al. Sanguisorba officinalis L synergistically enhanced 5-fluorouracil cytotoxicity in colorectal cancer cells by promoting a reactive oxygen species-mediated, mitochondriacaspase-dependent apoptotic pathway. Sci Rep. 2016;6:34245.

26. Peng JM, Zhang LX, Ma J, Guan ZB. The review of Amomum villosum in Xishuangbanna. Zhongguo Zhong Yao Za Zhi. 2006;31(2):97-1.

27. Wu Y, Ge F, Shi Q, Tan X, Wu H. Study of supercritical-CO2 fluid extraction in extracting essential oils of Amomun tsao-ko. Zhong Yao Cai. 1997;20(5):240-1.

\section{Submit your next manuscript to BioMed Central and we will help you at every step:}

- We accept pre-submission inquiries

- Our selector tool helps you to find the most relevant journal

- We provide round the clock customer support

- Convenient online submission

- Thorough peer review

- Inclusion in PubMed and all major indexing services

- Maximum visibility for your research

Submit your manuscript at www.biomedcentral.com/submit
) Biomed Central 